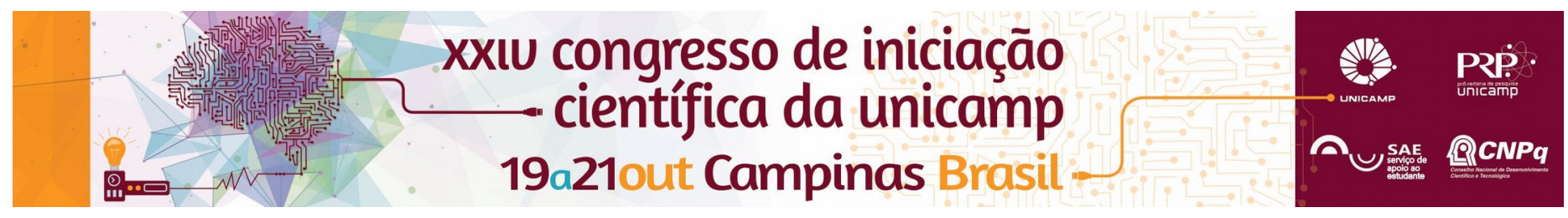

\title{
Mechanical engineering techniques applied to denture fixation analysis
}

\section{Thaís B. Baker*, Danny O. M. Marin, Pedro Y. Noritomi, Daniel T. Kemmoku, Marco A. Compagnoni, Ana C. Pero}

\begin{abstract}
Engineering software have been used to support healthcare researchers for a better understanding of the biomechanics. This work demonstrate the modeling process from CAD to FEA aiming to simulate three fixation implant sets with two different load cases on anatomically modeled reabsorbed mandible with overdenture in order to verify the different stress distributions in the bone and the displacement pattern of the prosthesis.
\end{abstract}

\section{Key words:}

Finite Element Analysis, Computed Aided Design, Bioengineering.

\section{Introduction}

Simplifications in the anatomical reconstruction and in the mechanical properties of living tissues are needed to enable in-silico experiments. Besides those considerations, FEA remains as a powerful tool to the development of new solutions and enhance the comprehension in biomechanics studies.

\section{Results and Discussion}

- Reverse engineering and CAD modeling

Every modeling is done on Rhinoceros ${ }^{\circledR}$ software. The bioCAD protocol is used to transform a ct scan of a reabsorbed mandible in a symmetric model. The overdenture prosthesis and implant sets are modeled based on three-dimensional scan and images.
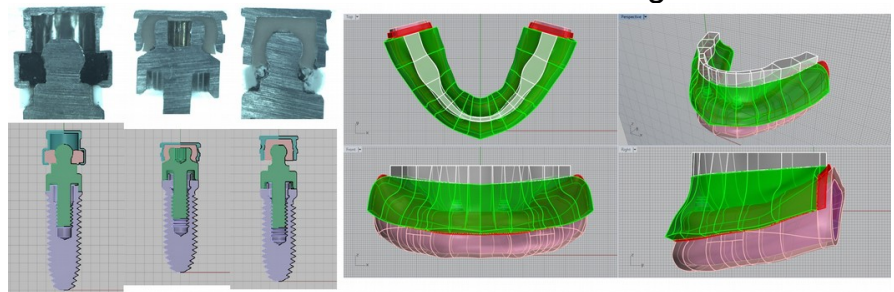

Image 1. From left to right: O-Ring, Equator and Mini Bola implant screw sets photos and models, next to one complete assembled model.

- Preprocessing

The assembly of the parts is imported to FEMAP $®$ software where the material is assumed linear and isotropic. The interaction between the prosthesis and the gingiva and between the implant pillar and it's polymer is considered sliding with the ability of separation. For all other parts, glued interaction is considered. The constraint is applied in the limits of modeled trabecular and cortical bones. Two different load cases applied a total of $100 \mathrm{~N}$, representing the bite acting on the first molar area. One dividing the force on both first molars and the other on only one side. The model is processed at NeiNastran®.

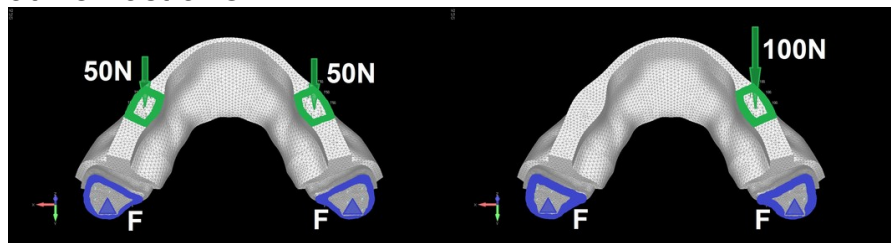

Image 2. Load cases from left to right: $50 \mathrm{~N}$ on both first molars and $100 \mathrm{~N}$ on one first molar.

\section{- Post processing}

Equator and O-Ring set of implants displayed, respectively, for both loads, the greatest and the lowest maximum principal stress in the bone, and the lowest and greatest displacement of the denture.
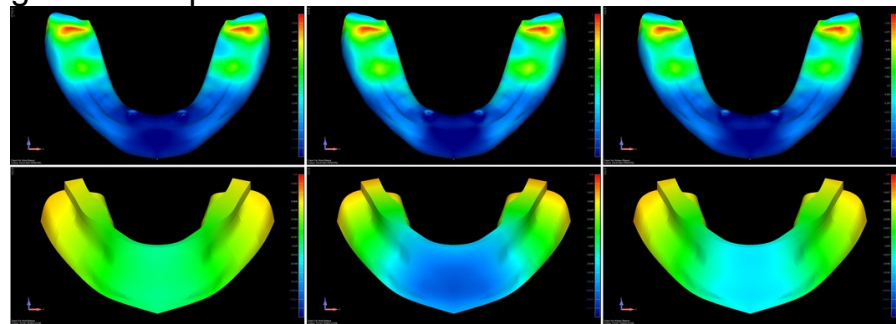

Image 3. Bilateral force results. Bone maximum principal stress (top line, scale form 0 to $1 \mathrm{MPa}$ ) and denture displacement (bottom line, scale from 0 to $0.05 \mathrm{~mm}$ ). From left to right: O-Ring, Equator and Mini Bola.
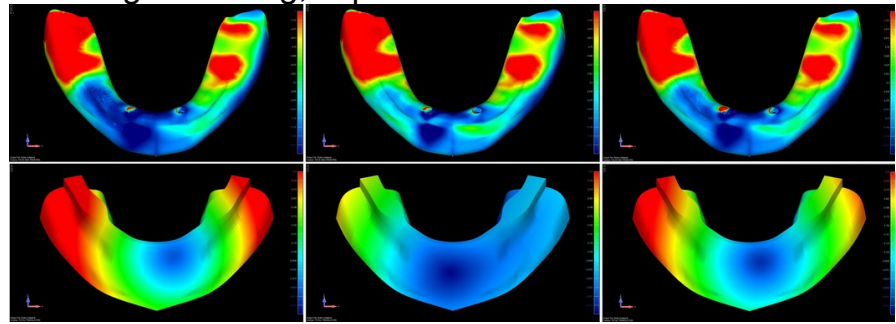

Image 4. Unilateral force results. Bone maximum principal stress (top line, scale form 0 to $1 \mathrm{MPa}$ ) and denture displacement (bottom line, scale from 0 to $0.25 \mathrm{~mm}$ ). From left to right: O-Ring, Equator and Mini Bola.

\section{Conclusions}

The stress distribution identify the critical regions and the displacement results show the stability of the connection sets. The combination of the simulation results with the clinical founds, enables the researcher to choose which treatment is most suitable for his purposes.

\section{Acknowledgement}

PIBIC/CNPq, CTI Renato Archer, DT3D members, Conexão and NeoDent - Grant N 0209/14

${ }^{1}$ KEMMOKU, Daniel Takanori et al. BioCAD techniques: Example on maxilla for rapid expansion simulation. In: BÁRTOLO, Paulo Jorge et al. Innovative Developments in Virtual and Physical Prototyping. Leiria, Portugal: Crc Press, 2011. Cap. 107. p. 715-718. 